\title{
Actitudes y conocimientos frente a la donación de órganos y tejidos
}

\section{Iris Tello-Rodriguez ${ }^{1}$}

RESUMEN: Objetivo: analizar la fuerza de asociación entre los conocimientos y la actitud hacia la donación y trasplante de órganos y tejidos en internos de enfermería de universidades privadas de Lima Norte. Metodología: el estudio fue de corte transversal. La muestra estuvo conformada por 82 internos de enfermería. Las variables del estudio fueron: conocimiento y actitud hacia la donación y trasplante de órganos y tejidos. Asimismo se incluyeron variables sociodemográficas. Se empleó el modelo de regresión logística ordinal donde se calcularon Odds Ratios (OR). Resultados: del total de los encuestados el $98 \%$ tenía un conocimiento medio sobre la donación y trasplante de órganos y tejidos y un $62.98 \%$ tenía una actitud indiferente. Tener un conocimiento alto (OR:11.21) se asoció con tener una actitud positiva hacia la donación y trasplante órganos y tejidos. Asimismo los factores disponibilidad de donar órganos y estudiar en una universidad privada católica se asociaron con una actitud positiva. Conclusiones: los internos de enfermería con un conocimiento alto sobre donación, con disponibilidad a donar órganos y tejidos y que estudian en una universidad privada católica tienen más posibilidades de presentar una actitud positiva. Estos resultados muestran una necesidad de políticas efectivas, de mayor educación con el fin de tener una aceptación y disponibilidad de donar órganos y tejidos cuando se es un donador potencial.

PALABRAS CLAVE: Obtención de Tejidos y Órganos; Actitud; Conocimiento; Enfermeros.

Citar como: Tello I. Actitudes y conocimientos frente a la donación de órganos y tejidos. CASUS. 2017;2(2):82-88. 


\section{INTRODUCCIÓN}

Un donador es aquella persona que con un gesto solidario, en vida o después de su muerte, consiente la disposición de sus órganos y tejidos. El trasplante mediante la transferencia de un individuo a otro en Perú es normado por la ley general de Donación y trasplante de órganos y/o Tejidos Humanos $N^{\circ} 28189$ (1).

Estudios realizados en España muestran buena aceptación hacia la donación y trasplante de órganos y tejidos. Esta puede mejorar aún ya que su fuente principal educativa es la televisión y los propios servicios de salud (2). España es líder mundial en la materia con el mejor índice de trasplantes por millón de población (p.m.p) tiene una tasa de 100.7 enfermos trasplantados p.m.p. El uso del modelo español con la Organización Nacional de Trasplante (ONT) de España genera la formación y la preparación sobre el tema (3). En América latina en el 2015 los pacientes trasplantados fueron en el caso de Argentina 40 p.m.p, Brasil 37.3 p.m.p, Uruguay 35 p.m.p, Colombia 25 p.m.p, Chile 17.9 p.m.p, Ecuador 8,2 p.m.p, Perú 6,3 p.m.p, Venezuela 6.2 p.m.p. En el caso de Perú aún se encuentra por debajo de los estándares ya que se realizan de manera inestable y no constante $(4,5)$.

Otro estudio en Estados Unidos tuvo una respuesta similar en relación a las nuevas técnicas de difusión de información (6). Estas deben ser accesibles para favorecer la buena aceptación del público que se siente inseguro, desconfiado y temeroso (6). Por otro lado, en Colombia se demuestra igualmente una buena actitud hacia la donación y trasplante de órganos, pero aún falta profundizar el conocimiento para que los miembros de la familia cumplan con el deseo del donador $(7,8)$. En el caso del Perú la donación se realiza basado en cómo figure en el Documento Nacional de Identidad (DNI) la opción de donador y posteriormente realizar el trámite de la cartilla en Seguro Social de Salud del Perú (EsSalud) para dejar constancia. No obstante, los familiares podrían negarse a realizar dicho procedimiento, ya sea por creencias o desconocimiento $(4,5)$.

La Organización Nacional de Donación y Trasplante (ONDT) informó que en el Perú hay un aproximado de 9 mil personas que necesitan un trasplante (9). En el 2014 la reducción de la tasa de donación de órganos fue de 2.2 por cada mil habitantes respecto a 4.1 en el 2013. Entre los trasplantes más frecuentes se encuentran el de córnea, riñón, hígado y corazón (9). El Registro Nacional de Identificación y Estado Civil documenta que de los 14580775 de peruanos solo el $14 \%$ portadores de DNI accede a donar sus órganos luego de fallecidos. Finalmente para nuestro país, la ONDT determinó que unas 300 personas mueren cada año debido a la falta de donantes de órganos, por una pobre cultura de donación, la creencia de mantener íntegro el cuerpo para su entierro y al temor existente en la población frente a la comercialización de órganos (10).

Factores como la falta de conocimiento han generado respuestas negativas o simplemente una indiferencia sobre la donación y trasplante de órganos entre la población impidiendo el aumento de donaciones para salvar sus vida o mejorar su calidad (4). Sin embargo, otros países con mayor desarrollo en el tema han demostrado que el conocimiento en el personal de salud sobre estos programas de donación y trasplante de órganos es un elemento que influye directamente en su éxito $(10,11)$. Este conocimiento sobre la donación de órganos puede estar influido por una serie de valores y principios que se inculcan desde que un enfermero se forma en una institución universitaria y contribuye a tener una actitud positiva (12). En este sentido son importantes las misiones y visiones de cada universidad $(13,14)$.

La disponibilidad de donar órganos es un gesto altruista y solidario hacia el prójimo (15). Por ello si se trabaja en un centro de salud, y si se tiene la disponibilidad de donar, aporta alta credibilidad en temas sanitarios a la población. Sin embargo, se verificó una carencia de formación adecuada sobre la donación y trasplante de órganos (16). Este es uno de los motivos que puede favorecer una actitud negativa hacia el tema tratado (16). En cuanto al sexo puede ser influyente en ser donante presentado una mayor predisposición el sexo femenino (17). A diferencia del sexo masculino que se muestra algo indiferente y, por ende, tiende a tener una actitud negativa (18).

A partir de la preocupante disminución de la tasa de donación en Perú, considerando la importancia que tiene el conocimiento en la disponibilidad hacia la misma y la necesidad de información al respecto se propuso como objetivo: analizar la relación entre el nivel de conocimiento y actitud hacia la donación y trasplante de órganos y tejidos. Este conocimiento es relevante pues permitirá proponer acciones con el fin de concientizar e incentivar la predisposición de los estudiantes de enfermería y buscar así disminuir esta problemática social (19).

\section{MATERIALES Y MÉTODOS}

El estudio fue correlacional y explicativo de corte transversal. La muestra estuvo conformada por 82 estudiantes que cursaban el internado hospitalario de dos universidades privadas de Lima Norte. E1 muestreo fue por censo para ambas universidades. Se incluyeron estudiantes de enfermería del décimo ciclo provenientes de dos universidades (una católica y otra no católica), que cursaban el internado hospitalario de 
Lima-Norte, mayores de 18 años a más, de ambos sexos. Se excluyeron estudiantes de otras carreras o que cursaban el internado comunitario.

La variable dependiente actitud hacia la donación y trasplante de órganos y tejidos se midió a través de un cuestionario tipo escala Likert adaptado de 22 preguntas validado a nivel nacional (20). Las actitudes fueron categorizadas según el criterio de estatinos los límites fueron: positivo (89 a 103), indiferente (72 a 88) y negativo (53 a 71).

Para medir la variable independiente conocimiento hacia la donación de trasplante de órganos y tejidos se empleó cuestionario de 14 preguntas igualmente validado a nivel nacional (20). Los conocimientos fueron categorizados, según el criterio de estatinos los límites fueron: alto (10 a 13), medio (7 a 9) y bajo (3 a 6).

Respecto a la variables sociodemográficas fueron consideradas, edad: variable categórica (20-25; 26-30; 31-35; 36-40); sexo: variable categórica (masculino y femenino); religión: variable categórica (católica, evangélica, otro, no tiene); procedencia: variable categórica (costa, sierra y selva); trabaja actualmente: variable categórica (sí, no); donante: definida con la siguiente pregunta ¿en su DNI figura como donante?: variable categórica (sí, no); disponibilidad: definida como la disponibilidad de donar órgano mediante la siguiente pregunta: ¿estarías dispuesto a donar un órgano? variable categórica (sí, no); tipo de universidad: variable categórica (católica, no católica).

Se elaboraron tablas de contingencia empleando el programa Stata 12. Para el análisis descriptivo se emplearon porcentajes y frecuencias. Respecto al análisis bivariado se utilizó la prueba estadística Chi2. Asimismo se empleó el modelo de regresión logística ordinal, donde se calcularon Odss Ratios (OR) e intervalos de confianza con una significancia estadística menor o igual a 0.05 .

El estudio contó con la aprobación de un comité de ética, asimismo se respetó la privacidad y confiabilidad de los datos de los encuestados.

\section{RESULTADOS}

De los 82 internos de enfermería un 62.2\% tenía una actitud indiferente hacia la donación y trasplante de órganos y tejidos. Un $60.98 \%$ del total de los encuestados tenía un conocimiento medio. La mayoría tenía entre 20 y 25 años $(67.07 \%)$, eran mujeres $(9.76 \%)$ y estarían dispuestos a donar y trasplantar sus órganos y tejidos $(73.17 \%)$. Por último un $53.66 \%$ pertenecían a una universidad no católica y un $59.76 \%$ trabajaba al momento de la encuesta (ver tabla 1).
Tabla 1. Características generales de los internos de enfermería

n

Actitud
Positiva
Indiferente
Negativa

\section{Conocimientos}

Alto

Medio

Bajo

\section{Edad}

$20-25$

$26-30$

$31-35$

$36-40$

17.1

8.5

7.3

\section{Sexo}

Femenino

90.2

Masculino

\section{Religión}

Católica

Evangélica

Otro

No tiene

\section{Procedencia}

Costa

68.3

Sierra

14.6

Selva

17.1

\section{Trabaja actualmente}

Sí

59.8

No

40.2

\section{Donante}

Sí 28

34.2

No

\section{Disponibilidad}

Sí

73.2

No

\section{Tipo de universidad}

No católica

Católica 
En la tabla 2 se observa que existe una asociación significativa entre el conocimiento sobre donación y trasplante de órganos y tejidos y la actitud positiva $(31.82 \%)$. Los factores ser mujer (18.92\%), trabajar actualmente $(18.37 \%)$, disponibilidad de donar órganos (21.67\%) y pertenecer a una universidad privada católica $(18.29 \%)$ se asociaron de manera significativa con una actitud positiva hacia la donación y trasplante de órganos. El resto de las variables no mostraron significancia estadística.

Tabla 2. Asociación descriptiva entre el conocimiento y sus factores sociodemográficos la donación y trasplante de órganos

\begin{tabular}{|c|c|c|c|c|c|c|c|c|}
\hline & $\begin{array}{c}\text { Negativa } \\
(\%)\end{array}$ & $\begin{array}{l}\text { Actitud } \\
\text { ndiferente } \\
(\%) \\
\end{array}$ & $\begin{array}{c}\text { Positiva } \\
(\%)\end{array}$ & p-value & $\begin{array}{l}\text { Tabla } 3 \text {. Fuerza de } \\
\text { actitud y sus facto } \\
\text { trasplante de órga }\end{array}$ & $\begin{array}{l}\text { Asocia } \\
\text { es hac } \\
\text { los }\end{array}$ & $\begin{array}{l}\text { ión entre la } \\
\text { a la donaci }\end{array}$ & ón y \\
\hline Conocimientos & & & & & & & & \\
\hline $\begin{array}{l}\text { Alto } \\
\text { Medio }\end{array}$ & $\begin{array}{c}4.5 \\
200\end{array}$ & 63.6 & 31.8 & 0.02 & & & Actitud & \\
\hline Bajo & 50.0 & $\begin{array}{l}60.0 \\
40.0\end{array}$ & $\begin{array}{l}14.0 \\
10.0\end{array}$ & & & OR & IC 95\% & p-value \\
\hline $\begin{array}{l}\text { Edad } \\
20-25\end{array}$ & & & & & Conocimiento & & & \\
\hline $\begin{array}{l}20-25 \\
26-30\end{array}$ & $\begin{array}{l}20.0 \\
14.3\end{array}$ & $\begin{array}{l}63.6 \\
64.3\end{array}$ & $\begin{array}{l}16.4 \\
21.4\end{array}$ & 0.94 & Bajo & 1.00 & - & - \\
\hline $31-35$ & 14.3 & 57.1 & 28.6 & & Medio & 3.54 & $0.87-14.42$ & 0.07 \\
\hline $36-40$ & & 50.0 & & & Alto & 11.21 & $2.24-56.00$ & 0.00 \\
\hline Sexo & & & & & & & & \\
\hline $\begin{array}{l}\text { Femenino } \\
\text { Masculino }\end{array}$ & $\begin{array}{l}14.9 \\
625\end{array}$ & 66.2 & $\begin{array}{l}18.9 \\
125\end{array}$ & 0.00 & Sexo & & & \\
\hline mascuinto & & & & & Femenino & 1.00 & - & - \\
\hline Religión & & & & & Masculino & 0.13 & $0.02-0.64$ & 0.01 \\
\hline $\begin{array}{l}\text { Católica } \\
\text { Evangélica }\end{array}$ & $\begin{array}{l}17.5 \\
33.3\end{array}$ & 63.5 & 19.0 & 0.77 & & & & \\
\hline Otro & 12.5 & $\begin{array}{l}50.0 \\
62.5\end{array}$ & $\begin{array}{l}16.1 \\
25.0\end{array}$ & & Trabaja actualmente & & & \\
\hline No tiene & & 60.0 & 0.0 & & Sí & 0.40 & $0.16-1.00$ & 0.05 \\
\hline Procedencia & & & & & No & 1.00 & - & - \\
\hline $\begin{array}{l}\text { Costa } \\
\text { Sierra }\end{array}$ & 16.1 & 66.1 & & 0.07 & & & & \\
\hline Selva & $\begin{array}{l}41.1 \\
14.3\end{array}$ & $\begin{array}{l}58.3 \\
50.0\end{array}$ & $\begin{array}{c}0.0 \\
35.7\end{array}$ & & Disponibilidad & & & \\
\hline & & & & & No & 1.00 & - & - \\
\hline $\begin{array}{l}\text { Trabaja } \\
\text { actualmente }\end{array}$ & & & & & $\mathrm{Si}$ & 2.48 & $1.27-9.56$ & 0.02 \\
\hline $\begin{array}{l}\text { Sí } \\
\text { No }\end{array}$ & 30.6 & 51.0 & 18.4 & 0.00 & & & & \\
\hline & 3.0 & 78.8 & 18.2 & & Tipo de universidad & & & \\
\hline Donante & & & & & No católica & 1.00 & ${ }^{-}$ & - \\
\hline $\begin{array}{l}\text { Sí } \\
\text { No }\end{array}$ & $\begin{array}{l}21.4 \\
18.5\end{array}$ & $\begin{array}{l}53.6 \\
66.4\end{array}$ & $\begin{array}{l}25.0 \\
14.8\end{array}$ & 0.44 & Católica & 2.98 & $1.17-7.57$ & 0.02 \\
\hline Disponibilidad & & & & & & & & \\
\hline $\begin{array}{l}\text { Sí } \\
\text { No }\end{array}$ & $\begin{array}{l}13.3 \\
36.4\end{array}$ & $\begin{array}{l}65.0 \\
54.6\end{array}$ & $\begin{array}{l}21.7 \\
9.1\end{array}$ & 0.04 & & & & \\
\hline Tipo de universic & & & & & & & & \\
\hline No católica & 25.0 & 65.9 & 9.1 & 0.04 & & & & \\
\hline Católica & 13.2 & 62.2 & 18.3 & & & & & \\
\hline
\end{tabular}

Tener un conocimiento alto influye en una actitud positiva hacia la donación y trasplante de órganos y tejidos (OR: 11.21; IC 95\%: 2.24-56.00). Los internos con disponibilidad al trasplante tienen 1.48 de probabilidad a tener un actitud positiva en comparación con los que no están disponibles (OR: 2.48; IC 95\%:1,27-9,56). Pertenecer a una universidad privada católica incide positivamente en tener una actitud positiva (OR: 2.98; IC 95\%: 1,17-7,57). Los que son hombres (OR: 0.13; IC 95\%:0,02-0,64) y trabajan actualmente (OR: 0.40; IC 95\%: 0.16-1.00) tienen menos posibilidades de tener una actitud positiva (ver tabla 3 ).

Tabla 3. Fuerza de Asociación entre la actitud y sus factores hacia la donación y trasplante de órganos 


\section{DISCUSIÓN}

En el estudio la mayoría de internos de enfermería tuvo un conocimiento medio y una actitud indiferente hacia la donación y trasplante de órganos y tejidos. Un conocimiento alto sobre la donación influye en tener una actitud positiva hacia la misma. Asimismo, hay asociación significativa entre los factores: disponibilidad de donar órganos y tejidos, pertenecer a una universidad católica y tener una actitud positiva. Sin embargo, el ser hombre con un trabajo tuvo menos posibilidad de presentar esta actitud.

El nivel de conocimiento bueno se asoció con tener una actitud positiva hacia la donación y trasplante de órganos y tejidos. Las personas que tienen conocimiento de la donación de órganos tienen una opinión favorable y una mayor disposición a donar órganos (13). Esto podría deberse a que si uno conoce los procedimientos, en qué momento se puede donar, que órganos se pueden donar y reconocer los requisitos para ser donador se pudieran tener menos objeciones a la misma $(10,21,22)$. Por otro lado, tener la disponibilidad de donar órganos se asoció con tener una actitud positiva hacia la donación y trasplante de órganos y tejidos. Esta disposición es importante pues es el primer eslabón para la toma de decisión de ser donante $(15,23,24)$. Igualmente pertenecer a una universidad privada católica se asoció con tener una actitud positiva hacia la donación y trasplante de órganos y tejidos. La literatura reporta que cualquier práctica religiosa y tener fe en Dios favorece la donación y trasplante de órganos y tejidos. La óptica religiosa considera este acto como un bien en favor de la humanidad $(13,14,25)$.
Ser de sexo masculino se asoció con tener una actitud negativa hacia la donación y trasplante de órganos. Los hombres tienen menos disposición para donar un órgano a diferencias de las mujeres (18). Sin embargo, el sexo femenino tiene mayor actitud positiva ya que tienen una mayor predisposición a ser partícipe por las relaciones sociales que crean en su entorno. Esto favorece la indagación, la adquisición de conocimientos y el desarrollo de una actitud positiva para ser donador (26).

Entre las limitaciones del estudio cabe mencionar que el cuestionario no fue diseñado para indagar en la falta de voluntad para ser donador. Se recomienda para futuros estudios incluir factores laborales y otras variables como la opinión de la familia respecto a la donación voluntaria.

\section{CONCLUSIONES}

Los internos de enfermería con un conocimiento alto sobre donación, con disponibilidad a donar y trasplantar órganos y tejidos que pertenecen a una universidad privada católica tienen más posibilidades de tener una actitud positiva. Sin embargo, los que tienen un trabajo actual y son hombres tienen menos posibilidades de tener esta actitud.

Un sistema de atención de salud que no conoce los factores que influyen en la donación y trasplante de órganos y tejidos fracasará en el desarrollo de buenas políticas y estrategias para la donación de órganos. Por ello, a partir de estos resultados, es necesario desarrollar nuevas políticas a través de intervenciones educativas para modificar conceptos erróneos y concientizar sobre la importancia de este acto voluntario.

\section{REFERENCIAS BIBLIOGRÁFICAS}

1. Ministerio de Salud [página principal en Internet]. Perú: Organización Nacional de Donación y Trasplante; c2010 [actualizado feb 2010; citado 27 nov 2015]. Disponible en: http://www.minsa.gob.pe/premio/arc hivos/boletin01.pdf

2. Abdo A, Leal G, Rocha M, Suárez J, Castellanos R, Ríos A. Conocimiento sobre la muerte encefálica y actitud hacia la donación de órganos en población no sanitaria de La Habana, Cuba. Invest Medicoquir. 2012;4(1):4-11.
3. Da Silva N, Schirmer J, De Aguiar B. Adaptación del Modelo español de gestión en trasplante para la mejora en la negativa familiar y mantenimiento del donante potencial. Texto Contexto Enferm.2011;20:59-65.

4. Ministerio de Sanidad, Servicios Sociales e Igualdad [página principal en Internet]. Madrid: Organización Nacional de Trasplantes; 2016 [actualizado 23 ago 2016; citado 03 ago 2017]. Disponible en: http://www.ont.es/Documents/23.08.2 016\%20NP\%20Datos\%20Registro\% 20Mundial\%20de\%20Trasplantes.pdf
5. Meza M. Informe final de capacitación en España sobre el proceso de donación y trasplante. Lima: Ministerio de Salud. Hospital Cayetano Heredia; 2011.

6. Volk M, Warren G, Anspach R, Couper M, Merion R, Ubel P. Attitudes of the American Public toward Organ Donation after Uncontrolled (Sudden) Cardiac Death. American Journal of $\mathrm{T} r$ a $\mathrm{n} \mathrm{s} \mathrm{p} 1$ a $\mathrm{n} \mathrm{t}$ a $\mathrm{t}$ i o n. 2010;10(3):675-680. 
7. Castañeda D, Alarcón F, Ovalle D, Martínez C, Gonzáles L, Burbano L, et al. Actitudes y creencias sobre la donación de órganos en Colombia: ¿Dónde se deben enfocar los esfuerzos para mejorar las tasas nacionales de donación? Rev Fac Med 2014;62(1):17-25.

8. Encolombia [página principal en Internet]. Bógota: Encolombia. Disponible en: https://encolombia.com/derecho/codi gos/civil-colombiano/codcivillibro1t2/

9. Organización Nacional de Donación y Trasplante [página principal en Internet]. Lima: MINSA; 2010 [citado 18 set 2015]. Disponible en http://www.minsa.gob.pe/ondt/situac ion.html

10. Tuesca R, Navarro E, Elles R, Espinosa D, et al. Conocimientos y actitudes de los médicos de instituciones hospitalarias en Barranquilla sobre donación y trasplante de órganos. Salud Uninorte. 2003;17:9-18.

11. Teixeira F, Almeida A, De Araújo K, Barbosa E. Perfil de los pacientes en la lista única de espera para trasplante cardíaco en el estado de Ceará. Arq Bras Cardiol 2010;95(1):79-84.

12. Universidad Católica Sedes Sapientiae [página principal en Internet]. Lima: Panizza L; [citado 18 julio 2017]. Disponible en: http://www.ucss.edu.pe/nosotros.htm 1

13. Calvanese N, Sanchez L, Redondo A, Milanés $\mathrm{C}$, et al. Factores psicosociales de la donación de órganos para trasplantes en Venezuela. Psicología y salud 2007;17(2):241-249.
14. Vélez E. Donación de órganos, una perspectiva antropológica. Rev Soc Enferm Nefrol. 2007;10(3):179-185.

15. Avilés L, Rivera M, Catoni M. Donar, un cambio de vida: comprender la experiencia de familiares que aceptaron la donación de órganos. Rev Med Chile. 2014;142:702-706.

16. Ríos A, López A, Ayala M, Sebastián M, Abdo A, Alán J, et al. Actitud hacia la donación de órganos del personal no sanitario de hospitales de España, México, Cuba y Costa Rica. Nefrología. 2013;33(5):699-708.

17. Frisancho A, Mesías T. Actitudes de los estudiantes de la Universidad Nacional Mayor de San Marcos hacia la donación de órganos. Investigación Educativa 2012;16(29):14-163.

18. Cruz S. Cuerpo, masculinidad y jóvenes. Iberóforum. Revista de Ciencias Sociales de la Universidad Iberoamericana. 2006;1(1):1-9.

19. Dos Santos M, Komatsu M, De Moraes E. Entrevista familiar no processo de doação de órgãos e tecidos para transplante. Acta Paul Enferm. 2012;25(5):788-94.

20. Callañaupa J. Relación entre el nivel de conocimientos y actitudes hacia la donación de órganos y tejidos de estudiantes de enfermería de la UNMSM, 2011 [tesis]. Lima: Universidad Nacional Mayor de San Marcos. Facultad de Medicina Humana; 2012.

21. Padilla J, Mora P, Monge A, Rodríguez R. Actitudes y conocimientos sobre la donación de órgano, trasplante y muerte cerebral en estudiantes de ciencias de la salud. Acta médica costarricense. 2015;57(4):179-183.
22. López A. Análisis de la actitud de los estudiantes de medicina hacia la donación de órganos para trasplante. [tesis doctoral]. Murcia: Universidad de Murcia. Departamento de Cirugía, Pediatría, Obstetricia y Ginecología; 2015.

23. Trasplante de órganos en el Perú. Lima; 16 de junio 2005. Lima: Academia Nacional de Medicina; 2006. p. 12-19.

24. Avendaño L, Alijama P, Arias M, Caramelo C, Egido J, Lamas S. Nefrología Clínica. $3^{\mathrm{a}}$ ed. Madrid: Editorial Panamericana; 2009.

25. Lima A, Silva J, Pereira L. Sufrimiento y contradicción: El significado de la muerte y del morir para los enfermeros que trabajan en el proceso de donación de órganos para trasplante. Enferm glob. 2009;15:1695-6141.

26. Ríos A, Conesa C, Ramírez P, San Martin A, Parrilla P ¿Está el personal de las unidades generadoras de donantes a favor de la donación de órganos de cadáver? Estudio en un hospital trasplantador. Med Clin. 2006;126(6):234-6. 


\section{Attitudes and knowledge regarding organ and tissue donation}

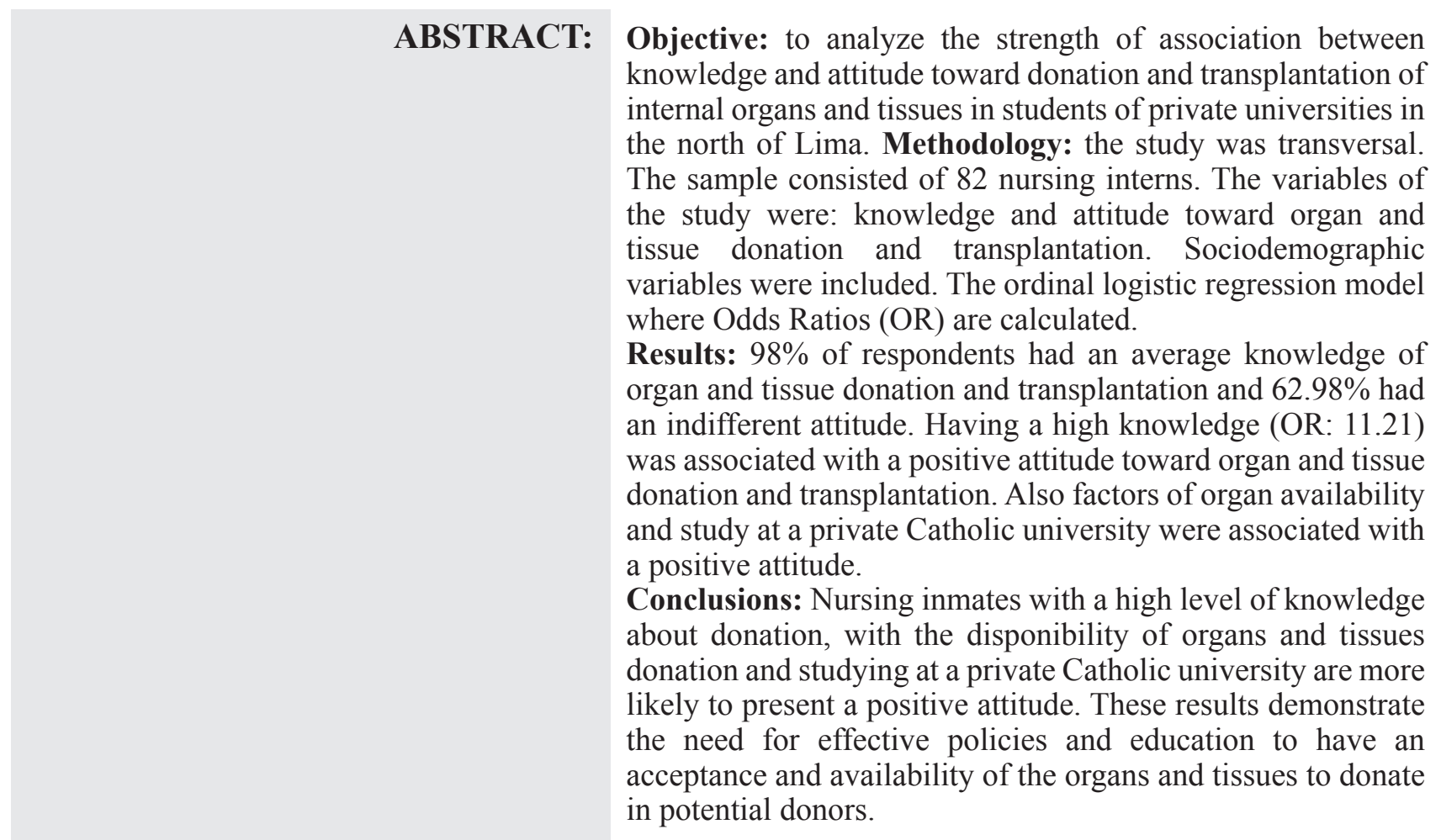

KEY WORDS: Tissue and Organ Procurement; Attitude, Knowledge; Nurses. 